\title{
ASSISTÊNCIA DE ENFERMAGEM NO CÂNCER DA MAMA E LINFONODO SENTINELA: UMA ABORDAGEM QUALITATIVA FACE À ATUAÇÃO DAS EQUIPES MULTIDISCIPLINARES
}

Elaine Cristina Carvalho Moura', Gerdane Celene Nunes de Carvalho², Grazielle Roberta Freitas da Silva ${ }^{3}$

${ }^{1}$ Mestre em Educação.

Enfermeira do Programa de Saúde da Família no Município de Picos, Piauí. ${ }^{3}$ Doutora em Enfermagem. Docente da Universidade Federal do Piauí, Brasil

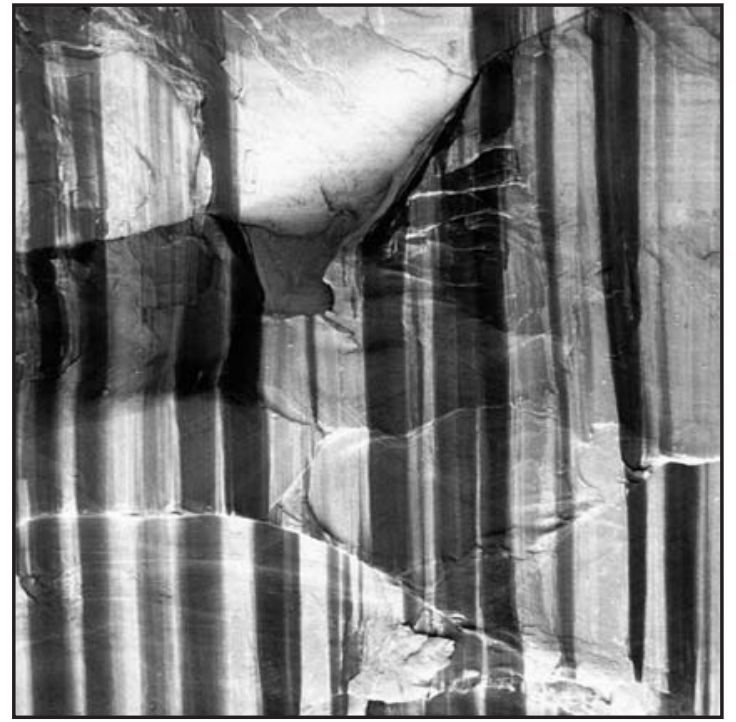

NURSERY CARE IN BREAST'S CANCER AND SENTINEL LYMPH NODE: A QUALITATIVE APPROACH TO HEALTH'S TEAM

\section{ABSTRACT}

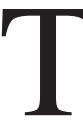
The present study aims analyzes the nursery care on health's team and the perspectives in the treatment of breast's Cancer by detection of the sentinel node. Treated of a descriptive qualitative research with data picked up through route semi structured applied to seven doctors and three nurses, chosen according to the professional experience in the thematic in focus, and discussed by the analyzes of content technique. The results point that this technology is constituted by stages and process that need the intervention of a health's team specifically demanded a nursing planning, updating and nursery care individual. Concluded that the sentinel node develops as qualitative change in the rehabilitation and women's recovery, deserving prominence the engagement of the health's team through which Knowledge are articulate in new Knowledge, resulting in benefits for the woman's health and, above all, opening a new area of actuation to Nurse "the nuclear nursing ".

Key words: Sentinel Lymph Node Biopsy; Breast's Cancer; nursery care; health's team.

ASISTENCIA DE ENFERMERÍA EN CÁNCER DE MAMA Y NÓDULO LINFÁTICO CENTINELA: UN ABORDAJE CUALITATIVO PARA EL EQUIPO DE SALUD

\section{RESUMEN}

71 estudio tiene como objetivo analizar la $\checkmark$ asistencia de enfermería en el equipo de salud y las perspectivas en tratamiento del cáncer de mama para la detección del nódulo linfático centinela. Se trata de una investigación cualitativa-descriptiva con datos recogidos por medio de encuestas semi-estructuradas aplicadas a siete doctores y tres enfermeras, escogidos según la experiencia profesional en el tema, y discutido por la técnica del análisis de contenidos. Los resultados apuntaron que esta tecnología se constituye por fases y procesos que necesita de la intervención de una equipo de salud, exigieron de la enfermería planear, actualizar e individualizar su asistencia. Así el Nódulo Linfático Centinela desarrolla como cambio cualitativo un cuadro de rehabilitación y recuperación, lo destacado es el compro- 
miso del equipo de salud para articular un nuevo conocimiento, produciendo beneficios para salud de la mujer y abriendo un nuevo campo de actuación para la enfermera "la enfermería nuclear."

Palabras clave: Biopsia del Nódulo Linfático Centinela; Cáncer de mama; enfermería; equipo de salud.

\section{RESUMO}

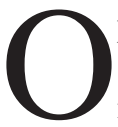
presente estudo tem como objetivo analisar a assistência de enfermagem em equipe multidisciplinar e as perspectivas no tratamento do câncer de mama pela detecção do linfonodo sentinela. Trata-se de uma pesquisa qualitativa descritiva com dados coletados por meio de roteiro semi estruturado aplicado a sete médicos e três enfermeiras, escolhidos conforme a experiência profissional na temática em foco, e discutidos pela técnica de análise de conteúdo. Os resultados apontam que esta tecnologia é constituída por etapas e processo que necessitam da intervenção multidisciplinar exigido especificamente da enfermagem planejamento, atualização e individualização da assistência. Conclui-se que o linfonodo sentinela evolui como mudança qualitativa no quadro de reabilitação e recuperação de mulheres, merecendo destaque o engajamento multidisciplinar em saúde por meio do qual saberes são articulados em novos saberes, resultando em benefícios para saúde da mulher e, sobretudo, abrindo um novo campo de atuação para o enfermeiro "a enfermagem nuclear".

Descritores: Biópsia de Linfonodo Sentinela; câncer de mama; enfermagem; equipe multidisciplinar.

\section{INTRODUÇÃO}

O tratamento do câncer de mama acarreta para a mulher uma série de conseqüências de ordem física e emocional. Diante disso, torna-se um desafio para a ciência buscar novos métodos que contribuam para seu aperfeiçoamento com o intuito de minimizar essas conseqüências sem, no entanto, colocar em risco a vida dessas pacientes.

Os tratamentos introduzidos para o câncer de mama durante séculos obedeceram a princípios
Halstedianos*. A partir de 1981, com os resultados dos estudos randomizados de Veronese, propôs-se novas abordagens de tratamento com preservação da mama. Atualmente, os estudos têm se direcionado para o status axilar (Vieira \& Pádua, 2000). Essa nova concepção de tratamento fundamenta-se nas recentes pesquisas do linfonodo sentinela que se refere ao "primeiro linfonodo a ser acometido locorregionalmente em pacientes com câncer de mama, evolui rapidamente e com perspectiva real de, em pouco tempo, poupar pacientes de uma linfadenectomia desnecessária" (Pereira, 2003).

Para Xavier et al (2002) a importância do estudo do linfonodo sentinela fundamenta-se na perspectiva de que o estadiamento patológico da axila apresenta um importante valor prognóstico quanto à sobrevida e traz informações necessárias que influenciam diretamente nas indicações terapêuticas, aumentando a cura clínica. Nas pacientes em que o diagnóstico é feito em fase mais precoce, a probabilidade de comprometimento axilar é cerca de $30 \%$ para aquelas pacientes com axila clinicamente negativa, portanto, $70 \%$ destas pacientes serão submetidas a linfadenectomia axilar desnecessariamente" (Vieira \& Pádua, 2000).

Em face da magnitude desta inovação técnicocientífica no tratamento do câncer de mama é necessário que a equipe de saúde busque conhecimentos que esclareçam as vantagens do tratamento para as pacientes, visto que, a equipe envolvida deve apreender a dimensão científica do tratamento para intervir conforme a sua área de atuação.

Nessa perspectiva, o profissional de enfermagem deve estar apto a planejar a assistência baseando-se no procedimento realizado e na percepção do mesmo pelas pacientes.

$\mathrm{O}$ presente estudo acrescenta conhecimento à área, na medida em que fundamenta uma perspectiva tecnológica para implementação de cuidados de enfermagem à mulher submetida à cirurgia por câncer de mama na equipe multidisciplinar. Nesse sentido, busca responder à seguinte inquietação: Qual a relação existente entre a detecção do linfonodo sentinela e a assistência planejada de enfermagem na equipe multidisciplinar? Objetiva analisar as perspectivas no tratamento do câncer de mama pela detecção do linfonodo sentinela e relacionar essa prática com a assistência de enferma- 
gem em equipe multidisciplinar. Parte-se da necessidade de "conhecer as indicações, limitações e reprodutibilidade desse novo método para sua aplicabilidade" (Galeb et al., 2000:110).

\section{PROCEDIMENTOS METODOLÓGICOS}

A investigação qualitativa descritiva que se dividiu em dois momentos: pesquisa bibliográfica e pesquisa de campo. e por fim uma pesquisa de campo. "A pesquisa bibliográfica é desenvolvida com base em material já elaborado, constituído principalmente de livros e artigos científicos" (Gil, 2002). A pesquisa documental baseou-se de fontes diversas voltadas para instituições privadas de saúde. A pesquisa de campo envolveu profissionais médicos e enfermeiros com experiências no tratamento do câncer de mama, utilizando a técnica do linfonodo sentinela. Foi realizada em Teresina, Piauí, onde o tratamento do câncer de mama pelo linfonodo sentinela era, à época, uma modalidade de tratamento exclusiva da rede privada de saúde.

Para a condução da pesquisa foram contempladas todas as normas da Resolução 196/96 do Conselho Nacional de Saúde, no qual foi apreciado e aprovado pelo Comitê de Ética em Pesquisa da Faculdade de Ciências Médicas - FACIME, localizado na cidade de Teresina-Piauí, sob número do protocolo 099/06, favorável a sua execução.

Os sujeitos da pesquisa foram sete médicos e três enfermeiros que atuam na modalidade de tratamento em estudo, totalizando dez sujeitos denominados respectivamente de M1 a M7 e E1 a E3. Dos médicos pesquisados, dois são nucleares, dois patologistas e quatro mastologistas. As enfermeiras atuam na área assistencial oncológica com experiência de três a quinze anos. Para efeito desse estudo, optou-se por priorizar os profissionais médicos e enfermeiras pela atuação nessa modalidade de tratamento.

Após a assinatura do Termo de Consentimento Livre e Esclarecido**, os sujeitos foram entrevistados no período de março a abril de 2007, por meio de entrevista semi-estruturada. Os roteiros de entrevista foram organizados para coletar dados que respondessem a questão norteadora do estudo. Tendo como questões comuns: Quais as mudanças necessárias na equipe de saúde com a implemen- tação da técnica do linfonodo sentinela? Quais os benefícios da identificação do linfonodo sentinela no tratamento do câncer de mama? As falas foram gravadas e transcritas com fidedignidade, resguardando, deste modo, o diálogo espontâneo.

Os dados foram tratados por meio da análise de conteúdo, seguindo-se do reconhecimento para a codificação, que originou a categoria intitulada: "Câncer de mama, linfonodo sentinela e a assistência de enfermagem: um olhar para a equipe multidisciplinar" com suas respectivas subcategorias: Tecnologia e linfonodo sentinela: processo e etapas; Enfermagem e detecção do linfonodo sentinela: novas perspectivas no cuidar; Equipe multiprofissional e multidisciplinaridade na atenção a clientes submetidas ao tratamento do câncer de mama com pesquisa do linfonodo sentinela.

\section{RESULTADOS E DISCUSSÃO}

Na subcategoria "Tecnologia e linfonodo sentinela: processo e etapas" referente a tecnologia implantada em câncer de mama na pesquisa do linfonodo sentinela no estado do Piauí obtive-se as seguintes respostas sobre o processo de detecção:

Para a realização da pesquisa do linfonodo sentinela a paciente precisa ter um tumor em fase inicial e axila clinicamente negativa.(M2, M3, M4, M5)

O mais utilizado para marcação do linfonodo sentinela é o material radioativo tecnésio. (M1, M2, M3, M4, M5, M6, M7)

Pode ser utilizado para marcação do linfonodo sentinela o azul patente, porém ele apresenta desvantagens... (M3, M6, M7) Outra maneira de fazer a pesquisa do linfonodo sentinela é fazer simultaneamente o tecnésio e o azul patente... (M3, M7)

Após a marcação é realizada a cirurgia para sua retirada e a análise pelo patologista... (M1, M2, M3, M4, M5, M6, M7)

Percebe-se nos depoimentos dos entrevistados sobre as etapas e processo de detecção do linfonodo sentinela como uma tecnologia confiável e com margem de segurança aceitável é necessário selecionar pacientes com tumores iniciais de mama e axila clinicamente negativa. Conforme outro depoimento: 


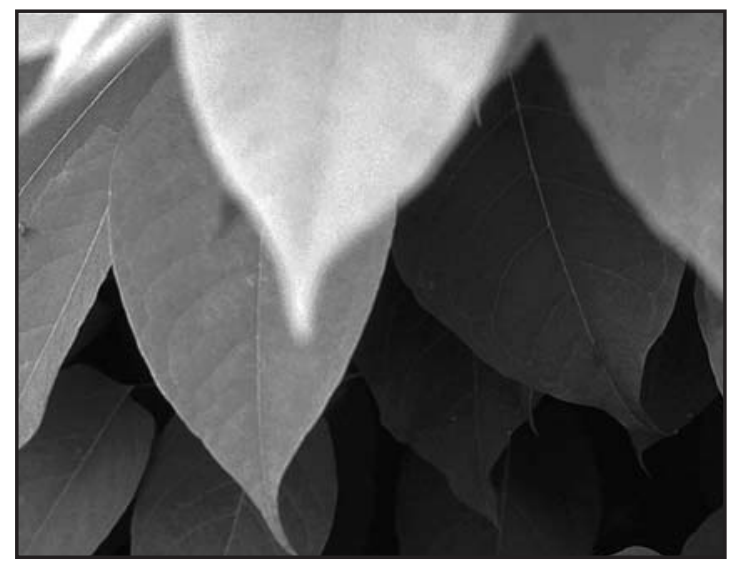

A pesquisa do linfonodo sentinela é realizado da seguinte maneira: você tem que selecionar as mulheres que tem indicação para fazer, são mulheres que tem lesões de mama menor que $3 \mathrm{~cm} \mathrm{e}$ axila clinicamente negativa (M4).

Nesta perspectiva, é possível inferir que para a indicação da pesquisa do linfonodo sentinela torna-se necessário o diagnóstico precoce do tumor de mama, já que sua evolução contra-indica a utilização por implicar negativamente em seus resultados.

Diante do mecanismo de funcionamento do sistema linfático deve-se considerar que o líquido formado por um tumor localizado na mama é disseminado para cadeia linfática na qual mantém ligação com o sistema linfático axilar. Assim torna-se indiscutível que a partir do momento que o líquido tumoral atingiu o linfonodo representa o inicio da metástase.

Partindo-se desta prerrogativa, a dissecção axilar foi por muito tempo prática comum para prevenir complicações do crescimento desordenado da mama tumoral. Essa imposição de tratamento ocorria devido à inexistência de avaliação de comprometimento linfonodal, dessa forma evitava-se que a metástase se propagasse, sem ao menos, ter a certeza de que ela tinha sido iniciada(Camargo \& Mark, 2000; Silva \& Zurrida, 2000).

Assim, faz-se exeqüível pela equipe de saúde a importância de incentivar o auto-exame das mamas com o intuito de tornar as mulheres os atores principais na identificação precoce do câncer de mama e conseqüentemente menos danos à sua saúde em decorrência da própria patologia e das complicações físicas e emocionais advindas de seu tratamento.

A técnica de detecção de linfonodo sentinela é um procedimento composto por etapas e processo pré-estabelecidos na prática clínica mundial, e por assim ser, vem se consolidando como prática clínica de escolha devido aos bons resultados, exigindo para sua realização profissionais especializados que dominem minuciosamente a técnica.

Os entrevistados descreveram três procedimentos realizados para a marcação do linfonodo sentinela. No entanto, apenas M6 e M7 fazem distinção entre os marcadores, azul patente e tecnésio, em termos de confiabilidade e acurácia. Tal fato decore principalmente da utilização quase exclusiva do tecnésio e da eventual utilização do azul patente. Nesse sentido M6 expõe:

A utilização do azul patente traz um problema técnico que é deixar os tecidos corados e isso em algumas situações dificulta o ato cirúrgico (M6)

Em decorrência dessas dificuldades ocorre menor adesão ao uso do azul patente, mesmo sendo uma técnica mais simples que a marcação pelo tecnésio, a chamada linfocintilografia. Conforme M7:

Você usa o azul patente massageia ali e espera uns 15 minutinhos e ele cora (M7).

O corante é injetado na região peritumoral da face axilar do tumor com a quantidade de 2 a $4 \mathrm{ml}$. Em contrapartida quando o linfonodo é marcado pelo tecnésio precisa de mais um profissional, o médico nuclear(Vieira \& Pádua, 2000; Pereira, 2003).

Fazendo um paralelo entre o referencial teórico e as falas dos profissionais entrevistados, um ponto que merece destaque é a baixa adesão na utilização do azul patente para marcação do linfonodo sentinela, fato este não contestado na sua utilização clínica mundial, sendo inclusive recomendado seu uso simultaneamente ao radiofármaco tecnésio, com o intuito de proporcionar uma maior confiabilidade aos resultados. Em contrapartida a literatura expõe que a combinação da linfocintilo- 
grafia através do detector gama probe e da orientação anatômica obtida pelo azul patente obtém-se as mais altas taxas de identificação do linfonodo sentinela (Xavier et al., 2002).

Desse modo, todo o processo de detecção descrito revela a utilização do linfonodo sentinela como uma perspectiva positiva no tratamento do câncer de mama, transformando essa nova tecnologia em mais uma alternativa para garantir melhor recuperação e mais qualidade de vida a mulheres acometidas.

Como efeito, a principal vantagem da pesquisa do linfonodo sentinela, é mostrada nas seguintes falas:

A principal vantagem é evitar a linfadenectomia desnecessária (M1, M4, M6),

Além do citado acima, os demais benefícios da pesquisa do linfonodo sentinela são conseqüência deste acontecimento, uma vez que suas complicações acarretam morbidades físicas e conseqüentes abalos psicológicos que podem afetar negativamente as clientes por longo período ou mesmo por toda a vida.

Segundo depoimento de M3:

Nem toda vez os linfonodos estavam comprometidos, mas a gente tirava todos os linfonodos de todo mundo e na prática 60 a $70 \%$ estavam fazendo uma cirurgia desnecessária. Com o linfonodo sentinela é diferente (M3).

Quando é realizada a pesquisa do linfonodo sentinela em clientes com câncer de mama e ele é diagnosticado como negativo para neoplasia, torna-se possível predizer que os demais linfonodos da cadeia não estão comprometidos. E desse modo, não há nenhuma necessidade de realizar o esvaziamento axilar (Mariani et al., 2001).

Porém, mesmo com tantos profissionais envolvidos e com vários estudos atestando veementemente por meio de pesquisas comparativas entre os métodos a efetividade de cada etapa para a detecção do linfonodo sentinela. Existem algumas situações que apresentam um resultado insatisfatório. Porém, tais resultados não implicam em sua limitação na prática, pois ao ser ponderado com os

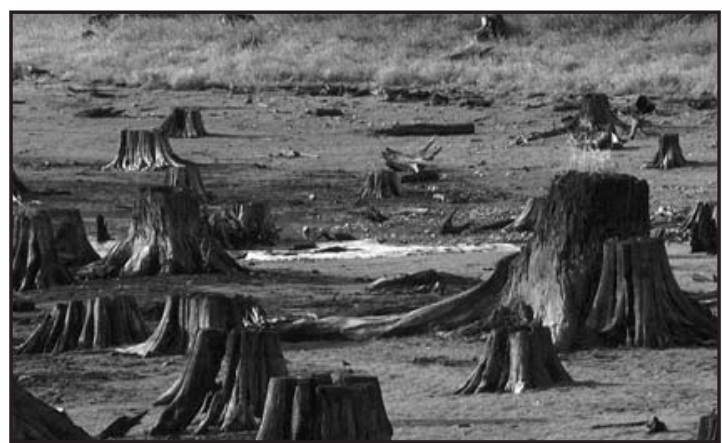

benefícios dessa técnica, tem sido observado um saldo geral positivo.

Os riscos do linfonodo sentinela existem como em qualquer cirurgia, mas eles são bem menores do que uma linfadenectomia axilar. Quando eu faço o linfonodo sentinela a cirurgia é menos extensa, é mais rápido e deixa menos seqüelas, é muito improvável algum comprometimento do movimento ou edema de braço (M3).

Nossa análise se focalizou no depoimento das enfermeiras, um novo olhar diante das vantagens da técnica em questão, focalizando preferencialmente as condições psicossocial das clientes, demonstrando assim o importante exercício interdisciplinar dos diversos profissionais envolvidos no processo:

Por tratar-se de uma técnica que permite reduzir as limitações físicas e psicológicas quando não houver necessidade de esvaziamento axilar, já que antes desta técnica várias pacientes podem ter sido submetidas ao esvaziamento axilar sem haver comprometimento dos linfonodos, é sem dúvida mais vantajoso ao paciente (E2).

Pelo exposto infere-se que os profissionais médicos focalizaram aspectos clínicos, preferencialmente, descrevendo as vantagens em termos de técnica cirúrgica, complicações evitadas e outros aspectos, tratando do procedimento em si e o que ele proporciona para a medicina na atualidade. As enfermeiras, em contrapartida, abordaram as vantagens advindas da implementação da técnica tanto em aspectos clínicos, quanto no conforto que ele proporciona à cliente, numa visão holística na 
medida em que descrevem sentimentos de tranqüilidade e auto-estima.

Ainda em relação às vantagens da técnica, infere-se que os profissionais médicos focalizaram aspectos clínicos, descrevendo as vantagens em termos de técnica cirúrgica e complicações evitadas, tratando do procedimento em si e o que ele possibilita para a medicina na atualidade. Enquanto as enfermeiras abordaram as vantagens advindas da implementação da técnica tanto em aspectos clínicos, quanto ao conforto que ela proporciona a paciente.

A subcategoria "Enfermagem e a detecção do linfonodo sentinela: novas perspectivas do cuidar" estabelece os parâmetros para o planejamento da assistência de enfermagem diante da tecnologia do linfonodo sentinela, dando origem ao seguinte agrupamento de falas:

Orienta o tratamento e o prognóstico, não há uma Sistematização da Assistência de Enfermagem-SAE concreta, pois os funcionários desconhecem o procedimento (E2)

Melhora a qualidade de vidas das pacientes I ... I não existe uma assistência pré-operatória, porque ela faz a verificação do linfonodo sentinela como tratamento externo (E1)

A paciente que faz o esvaziamento axilar requer muito mais cuidado que aquela que não fez. (E3)

Partindo-se dos depoimentos a atuação do enfermeiro no tratamento do câncer de mama contempla uma visão diferenciada da cliente, quando realiza a detecção do linfonodo sentinela. Neste sentido, é considerado que quando não existe indicação para uma linfadenectomia, alguns cuidados são dispensados e isso faz com que a paciente tenha uma menor possibilidade de desenvolver infecção e outras intercorrências. Conforme E3:

Quando a mulher se submete a uma linfadenectomia, ela tem estímulos que determinam o futuro dela como mulher e como paciente, por isso deve ser prestada uma série de cuidados (E3).

Neste enfoque, concentra-se a maior importância da introdução da pesquisa do linfonodo sentinela na prática clínica. Quando o status axilar é preservado, a assistência de enfermagem não fica tão direcionada as práticas que pretendem prevenir o linfedema no membro superior (Mariani et al., 2001). Contudo, isso não exime a atuação da enfermagem quanto ao auto cuidado e auto-estima da cliente focalizando além dos cuidados perioperatórios a educação em saúde.

No entanto, as enfermeiras entrevistadas declararam que a assistência de enfermagem tem se restringido aos cuidados em âmbito hospitalar não alcançando as etapas e processos de preparação que muitas vezes ocorrem em outros espaços de saúde, como clínicas nucleares, impossibilitando assim uma assistência sistematizada e continuada.

Vale ressaltar, que segundo DellAcqua. \& Miyadahira(2002), a importância da implantação e implementação da SAE a cliente em foco, a fim de proporcionar um acompanhamento continuado e fortalecer a incorporação da prática sistematizada da assistência em enfermagem como possibilidade relevante de cumprir as bases científicas e cognitivas do cuidar da enfermagem. E1 comenta que:

Infelizmente não existe essa assistência préoperatória, porque a paciente faz todo preparo com um tratamento externo. Ela já vem com a medicação feita da Clínica Nuclear, já vem preparado para o centro cirúrgico, quando chega na enfermaria já fez a ressecção do linfonodo sentinela e a mastectomia, aí não tem como a gente fazer esse preparo antes (E1).

A realização da pesquisa do linfonodo sentinela em clientes com câncer de mama requer, portanto a assistência de enfermagem no perioperatório, contemplando todas as etapas e processos da técnica, principalmente por se tratar de um procedimento desconhecido e até estranho as clientes que o realizam(Pereira, 2003; Mariani et al, 2001). A enfermagem deve está apta a fomentar informações de forma contínua e individualizada, de forma a esclarecer em que consiste a técnica, manifestações e benefícios no tratamento e recuperação da cliente.

Com a técnica do linfonodo sentinela pode-se evitar mutilações desnecessárias. Segundo estudo realizado na cidade de Salvador, estado da Bahia, no Brasil(Azevedo \& Lopes, 2006), a mastecto- 
mia, como uma das abordagens terapêuticas indicadas, representa uma ameaça à vida da mulher devido à mutilação de um órgão que representa a sua feminilidade.

$\mathrm{Na}$ subcategoria "Equipe multiprofissional e multidisciplinaridade na atenção a clientes submetidas ao tratamento do câncer de mama com pesquisa do linfonodo sentinela" tratou-se das mudanças necessárias na equipe multiprofissional em saúde com a implementação da pesquisa do linfonodo sentinela, obtivemos as seguintes respostas:

O que o profissional precisa e aderir e se habituar (M1)

Três profissionais são necessário, o primeiro o médico nuclear, o segundo o mastologista e o terceiro o patologista (M2, M3, M4, M5, M6, M7)

Depende de uma boa interação entre o mastologista, o fisioterapeuta e a equipe de enfermagem (M5,E2)

Integralidade, multidisciplinar, tem que ter comunicação (E1)

Precisa de médicos, do cuidado da enfermagem, nutrição adequada e psicólogo (E3)

As falas revelaram que $15 \%$ dos médicos indicam a necessidade dos profissionais conhecerem a técnica e aplicá-la. Em 85\% dos depoimentos dos médicos foi relatada ainda a necessidade de um médico nuclear, do mastologista e do patologista. Indicando também por $15 \%$ dos médicos e $33 \%$ dos enfermeiros a necessidade de uma boa interação entre o mastologista, o fisioterapeuta e a equipe de enfermagem, além dos cuidados gerais de enfermagem, médicos, nutricionais e psicológicos.

Considerando o depoimento dos entrevistados, verificou-se a importância que enfermeiros e médicos atribuem para a articulação do trabalho em equipe numa visão multiprofissional, embora com diferentes olhares quanto às necessidades da clientela. E3 explica a conotação multiprofissional ao dizer que:

É necessário que a equipe seja realmente uma equipe multiprofissional, pois a cliente precisa do médico, precisa do cuidado da assistência de enfermagem, precisa da nutrição adequada, precisa do psicólogo. O que eu acho mais importante é que essa equipe nunca deixe de ser uma equipe multiprofissional. Eu acho que a cliente deve ser assistida de uma maneira holística (E3).

Vale ressaltar que dentre os profissionais médicos foi destacado a importância da categoria nas diversas etapas do procedimento de detecção do linfonodo sentinela, como a inserção da especialista em medicina nuclear para realizar a linfocintilografia; o mastologista para realizar a extração do linfonodo sentinela, a mastectomia e a linfadenectomia, se indicada; e o patologista para proceder ao estudo minucioso do linfonodo sentinela, numa visão ampliada e definida do curar conforme a contribuição de várias especialidades médicas. M3 reforça essa visão:

A cirurgia é mais elaborada, é preciso que inclua o médico nuclear e o patologista, além do mastologista (M3).

Os enfermeiros, no entanto, apontaram uma perspectiva de equipe multidisciplinar, que se refere à união de saberes da equipe com intuito de prestar para cliente uma assistência que se destina a sua recuperação global, a fim de percebê-la numa dimensão biopsicosocioespiritual contando com a necessidade multidisciplinar dos profissionais alicerçada no diálogo.

Isso ocorre porque atualmente o trabalho em saúde tem adotado uma assistência holística, ou seja, o indivíduo é assistido como um todo. Deste modo, torna-se necessária uma abordagem totalizadora para o alcance dos objetivos de promover saúde, entendida como qualidade de vida. Nesta concepção, as várias categorias devem trabalhar em conjunto, embora preservando espaços para a aplicação do núcleo de conhecimento de cada uma delas, exigindo, no entanto, a comunicação em todas as situações para contemplarem uma abordagem multidisciplinar (Ducan, Schmidh \& Guiliane, 2004).

É importante, diante dos depoimentos esclarecer a diferença essencial entre uma equipe multiprofissional e multidisciplinar. Quando o trabalho da equipe se baseia no conhecimento cientifico racionalista de cada categoria profissional ocorre uma compartimentalização, de forma que o trabalho se desenvolve paralelamente, havendo pouca ou nenhuma discussão e elaboração das categorias 
entre sim, resultando em uma atenção fragmentada aos pacientes, trata-se de uma equipe predominantemente multiprofissional.

Por outro lado, quando as várias categorias trabalham em conjunto, num processo habitual e cotidiano de uma práxis construída por uma equipe que estabelece relações entre os saberes dos vários profissionais a fim de incorporar os olhares de intersecção na atuação de todos os profissionais envolvidos em prol da saúde dos clientes, tem-se a dimensão multidisciplinar.

\section{CONCLUSÃO}

A técnica do linfonodo sentinela, na prática clínica, representa uma nova tecnologia em saúde, à medida que proporciona inúmeras vantagens para o tratamento de mulheres submetidas à cirurgia por câncer de mama, desde a seleção das pacientes para o procedimento até a retirada e análise do linfonodo no momento da cirurgia pelo patologista. Portanto, a pesquisa aponta melhor recuperação física e psicossocial, visto que, no tratamento cirúrgico se preserva a região axilar sem o comprometimento de todos os linfonodos, resultando em melhor qualidade de vida.

Para tanto, é necessária a atuação de uma equipe muiltiprofissional que alcance uma abordagem multidisciplinar, uma vez que a técnica exige mais de uma especialidade médica no acompanhamento e execução do tratamento, uma assistência de enfermagem sistematizada e a comunicação entre os diversos profissionais de saúde. Cabe aos profissionais encontrar o ponto de interação em busca da efetivação dessa prática.

Nesse sentido, a enfermagem necessita se apropriar dessa tecnologia, na perspectiva de visualizar um novo campo de atuação "a enfermagem nuclear", pois o tratamento nuclear vem se mostrando uma realidade na prática clínica mundial, trazendo inúmeros benéficos aos pacientes na área de saúde.

Assim, o fortalecimento da SAE durante as etapas de execução da técnica do linfonodo sentinela por todo o perioperatório, pode enriquecer as necessárias discussões multidisciplinares frente à tecnologia proposta, caracterizando, desse modo, mais do que uma integração muiltiprofissional, e sim uma experiência multidisciplinar na área hospitalar.

\section{REFERÊNCIAS}

- Azevedo, R.; Lopes, R.(2006). Experience of breast cancer diagnosis and radical mastectomy: a phenomenological study. Online Brazilian Journal of Nursing[Online] 5:1. Available: http://www.uff.br/objnursing/index.php/nursing/article/view/ 137 .

- Camargo, M.C.; Mark, Â.G.(2000) Reabilitação Física no Câncer de Mama. São Paulo (SP): Roca.

- DellAcqua, M.C.Q.; Miyadahira, A.M.K. (2002) Ensino do processo de enfermagem nas escolas de graduação em enfermagem do estado de São Paulo. Rev Latino-am Enfermagem. 10(2):185-91.

- Ducan, B.B.; Schmidh, M.I.; Guiliane, E.R. (2004) Medicina ambulatorial: conduta de atenção primária baseada em evidências. Porto Alegre (RS): Artmed.

- Galeb, N.A.; Garrido, M.M.; De Luca, L.A.; Osório, C.A.B.T.; Costa, R.L.R.; Gamaeiro, P.L. et al.(2000) Estudos das técnicas para pesquisa do linfonodo sentinela no câncer de mama. Rev Brasileira de Mastologia.10(3):107-114.

- Gil, A.C.(2002). Como elaborar projetos de pesquisa. São Paulo (SP): Atlas.

- Minayo, C.S.(1999) Pesquisa social: teoria, método e criatividade. Petrópolis (RJ): Vozes.

- Mariani, G.; Moresco, L.; Viale, G., Villa, G.; Bagnasco, M.; Paganelli, G.; et al. (2001) Radioguided Sentinel Lymph Node Biopsy in Breast Cancer Sugery. Journal of Nuclear Medicine.42(8):1198-1215.

- Silva, O.E.; Zurrida, S.(2000) Câncer de mama: Um guia para médicos. São Paulo (SP): Atlanta.

- Pereira, P.M.S.(2003). Linfonodo Sentinela: presente e futuro. Jornal do Clube da Mama [série da Internet] 2003 Jan [citado 2008 out 31]; 5(1): [6p]. Available: www.clubedamama. org.br?materiais.php?ação=ler\&codigo $=63$.

- Vieira, S.C.; Pádua, F.A.F. (2000) Linfonodo Sentinela no Câncer de mama. Rev Médica do Hospital São Marcos; 4(1):24-26.

- Xavier, N. L, Amaral, B.B, Spiro, B.L.; Almeida, M.J.; Menke, C.H.; Biazú, J.V. et al.(2002) Detecção do linfonodo Sentinela em Câncer de Mama: Comparação entre Métodos. Rev Brasileira de Ginecologia e Obstetrícia 24(7):479-484.

* Foi consagrado desde 1882 por Willians Hasted como tratamento cirúrgico da mama a linfadenectomia axilar associada à mastectomia e ressecção dos músculos peitorais.

** O termo de Consentimento Livre e Esclarecido foi assinado por todos os sujeitos estudados em consonância com o disposto no Decreto no 93933 de 14 de janeiro de 1987 na Resolução 196/1996 do Conselho Nacional de Saúde que dispõe sobre as Diretrizes e Normas Regulamentadoras de Pesquisa envolvendo Seres Humanos. Na perspectiva de assegurar ao sujeito, livre de qualquer coerção, o direito de autorizar o uso de seus depoimentos no estudo e até mesmo de retirar-se deste a qualquer tempo, caracterizando a voluntariedade do indivíduo em colaborar com pesquisas. 